\title{
Overcoming Macrophage-Mediated Axonal Dieback Following CNS Injury
}

\author{
Sarah A. Busch, ${ }^{1 \star}$ Kevin P. Horn, ${ }^{1 \star}$ Daniel J. Silver, ${ }^{2}$ and Jerry Silver ${ }^{1}$ \\ ${ }^{1}$ Department of Neurosciences, Case Western Reserve University, Cleveland, Ohio 44106, and ${ }^{2}$ Department of Neuroscience, College of Medicine, University \\ of Florida, Gainesville, Florida 32611
}

\begin{abstract}
Trauma to the adult CNS initiates multiple processes including primary and secondary axotomy, inflammation, and glial scar formation that have devastating effects on neuronal regeneration. After spinal cord injury, the infiltration of phagocytic macrophages coincides with long-distance axonal retraction from the initial site of injury, a deleterious phenomenon known as axonal dieback. We have previously shown that activated macrophages directly induce long-distance retraction of dystrophic axons in an in vitro model of the glial scar. We hypothesized that treatments that are primarily thought to increase neuronal regeneration following spinal cord injury may in fact derive a portion of their beneficial effects from inhibition of macrophage-mediated axonal retraction. We analyzed the effects of protease inhibition, substrate modification, and neuronal preconditioning on macrophage-axon interactions using our established in vitro model. General inhibition of matrix metalloproteinases and specific inhibition of MMP-9 prevented macrophage-induced axonal retraction despite significant physical interactions between the two cell types, whereas inhibition of MMP- 2 had no effect. Chondroitinase $\mathrm{ABC}$-mediated digestion of the aggrecan substrate also prevented macrophage-induced axonal retraction in the presence of extensive macrophage-axon interactions. The use of a conditioning lesion to stimulate intrinsic neuronal growth potential in the absence of substrate modification likewise prevented macrophage-induced axonal retraction in vitro and in vivo following spinal cord injury. These data provide valuable insight into the cellular and molecular mechanisms underlying macrophage-mediated axonal retraction and demonstrate modifications that can alleviate the detrimental effects of this unfavorable phenomenon on the postlesion CNS.
\end{abstract}

\section{Introduction}

Regeneration in the adult CNS is unsuccessful as a result of the decreased intrinsic regenerative capacity of affected neurons (Neumann and Woolf, 1999), myelin-associated inhibitory factors (Filbin, 2003), and components of the glial scar (Busch and Silver, 2007). After spinal cord injury, infiltration of activated macrophages correlates with long-distance retraction of dystrophic endbulbs, a deleterious phenomenon known as axonal dieback (Horn et al., 2008). Additionally, macrophages have the capacity to induce extensive retraction of dystrophic neurons following direct physical interactions in vitro. Here, we sought to investigate targets for modification of these interactions that could ameliorate axonal dieback. We hypothesized that several treatments primarily thought to increase neuronal regeneration derive a portion of their beneficial effects from inhibition of macrophage-mediated axonal retraction. We examined the effects of macrophage-associated protease inhibition, treatment of the substrate with chondroitinase $\mathrm{ABC}(\mathrm{ChABC})$, and stimula-

\footnotetext{
Received Jan. 28, 2009; revised April 30, 2009; accepted June 16, 2009.

This work was supported by National Institutes of Health-National Institute of Neurological Disorders and Stroke Grant NS25713 (J.S.), the Brumagin-Nelson Fund for Spinal Cord Injury Research, and the National Institutes of Health Training Grant T32 AG00271. We extend our thanks to Hongmei Hu and Fernando X. Cuascut for technical assistance and to Maryanne Pendergast for assistance with imaging.

*S.A.B. and K.P.H. contributed equally to this work.

Correspondence should be addressed to Dr. Jerry Silver, Department of Neurosciences, Case Western Reserve

University, 2109 Adelbert Road, S0M E-658, Cleveland, Ohio 44106. E-mail: jxs10@cwru.edu.

D0I:10.1523/JNEUROSCI.1151-09.2009

Copyright $\odot 2009$ Society for Neuroscience $\quad 0270-6474 / 09 / 299967-10 \$ 15.00 / 0$
}

tion of intrinsic neuronal growth capacity with a conditioning lesion (CL) on macrophage-neuron interactions in an in vitro model of the glial scar. We then sought to determine the effects of CL on macrophage-mediated axonal retraction in vivo.

The matrix metalloproteinases (MMPs) are a family of zincdependent proteinases that can degrade components of the extracellular matrix (ECM) and cleave cell surface receptors and adhesion molecules (Yong, 2005). The upregulation of MMPs after CNS injury can be beneficial or detrimental depending on the length of time after the injury, the profile of inflammatory cells at the injury site, and the substrates present (Popovich and Longbrake, 2008). Profuse expression of MMPs leads to demyelination and axonal injury (Yong et al., 2001), and current research focuses on potential therapeutics that attenuate excess MMP activity after CNS injury (Noble et al., 2002).

Chondroitin sulfate proteoglycans (CSPGs) are potently inhibitory ECM molecules that contribute to limited recovery via restriction of regeneration and plasticity after CNS injury (Silver and Miller, 2004; Yiu and He, 2006). The barrier formed by CSPGs induces a dystrophic state in growth cones as they enter the lesion site (Davies et al., 1999). Use of the enzyme ChABC, which cleaves the inhibitory glycosaminoglycans from the protein core of CSPGs, has been shown to promote axon regrowth and plasticity after injury (Bradbury et al., 2002; Steinmetz et al., 2005; Houle et al., 2006; Tester and Howland, 2008).

A substantial amount of research suggests that increasing the intrinsic growth potential of damaged sensory neurons enables them to overcome inhibition in the injured CNS (Qiu et al., 2002; 
Neumann et al., 2005). A CL fosters regeneration of adult DRG neurons after subsequent injury to their central axons (Neumann and Woolf, 1999). While the exact sequence of events leading to the CL phenomenon is unknown, the effect is likely due to changes in expression of regeneration-associated genes (Seijffers et al., 2007).

Our results suggest that macrophagemediated axonal dieback is extremely complex and can be prevented in a number of ways. Here we demonstrate that inhibition of MMP-9, treatment with ChABC, and administration of a CL all enable neurons to successfully overcome macrophage-mediated axonal retraction.

\section{Materials and Methods}

Time-lapse dish preparation. Delta-T cell culture dishes were prepared as previously described (Horn et al., 2008). Briefly, a number 2 bit was used to drill a single hole through the upper half of each dish to allow for the addition of enzymes or inhibitors to the cultures during time-lapse microscopy. Culture dishes were rinsed with sterile water and then coated with poly-L-lysine $(0.1 \mathrm{mg} / \mathrm{ml}$, SigmaAldrich) overnight at room temperature, rinsed with sterile water, and allowed to dry. Aggrecan spot gradients were created by pipetting $2.0 \mu \mathrm{l}$ of aggrecan solution $(2.0 \mathrm{mg} / \mathrm{ml}$, Sigma-Aldrich) in calcium- and magnesiumfree HBSS (HBSS-CMF, Invitrogen) onto the culture surface. Six spots were placed per dish and when the aggrecan spots were dry, the entire surface of the dish was bathed in laminin solution $(10 \mu \mathrm{g} / \mathrm{ml}$; BTI $)$ in HBSS-CMF for $3 \mathrm{~h}$ at $37^{\circ} \mathrm{C}$. The laminin bath was removed and cells were plated immediately without allowing the dish surface to dry.

$D R G$ dissociation. DRGs were harvested as previously described (Tom et al., 2004). Briefly, DRGs were gently dissected from adult female Sprague Dawley rats (Harlan) or embryonic day 16 rats and incubated in a solution of Collagenase II (200 $\mathrm{U} / \mathrm{ml}$, Worthington Biochemical) and Dispase II (2.5 U/ml, Roche Diagnostics) in HBSS. Cells were centrifuged at a low speed, washed, and gently triturated in HBSS-CMF three times. The dissociated DRGs were then resuspended in Neurobasal-A (for adult neurons) or Neurobasal (for embryonic neurons) media supplemented with B-27, GlutaMax, and penicillin/ streptomycin (Invitrogen) and counted. DRGs were plated on Delta-T dishes (Fisher) at a density of 6000 cells/dish in $2 \mathrm{ml}$ of media.

Cell line macrophage cultures. NR8383 cells, an adult Sprague Dawley alveolar macrophage cell line (ATCC \# CRL-2192, American Type Culture Collection), were cultured as described previously (Yin et al., 2003). Briefly, cells were cultured in F-12K media (Invitrogen) supplemented with 15\% FBS, GlutaMax, penicillin/streptomycin (Invitrogen), and sodium bicarbonate (Sigma-Aldrich) using uncoated tissue culture flasks (Corning). Macrophages were prepared for time-lapse microscopy experiments as described previously (Horn et al., 2008). Briefly, cells were harvested with $0.5 \%$ trypsin/EDTA (Sigma-Aldrich), washed three times with HBSS-CMF, and plated in uncoated tissue culture flasks at a density of $1.0 \times 10^{6} / \mathrm{ml}$ in serum-free F-12K. Before addition to time-lapse dishes, macrophages were harvested with EDTA and a cell scraper and resuspended at a density of $2.5 \times 10^{5} / 70 \mu \mathrm{l}$ in Neurobasal-A supplemented as above with the addition of HEPES (1:500; $50 \mu \mathrm{M}$, Sigma-Aldrich). Macrophages were stained for ED-1 (Millipore Bioscience Research Reagents) and MMP-9 (1: 500; Millipore).

Time-lapse microscopy studies. Adult and embryonic DRG neurons were incubated at $37^{\circ} \mathrm{C}$ for $48 \mathrm{~h}$ before time-lapse imaging. Neurobasal-A (adult neurons) or Neurobasal (embryonic neurons) media with HEPES (50 $\mu \mathrm{M}$, Sigma-Aldrich) was added to the cultures before transfer to a heated stage apparatus. Time-lapse images were acquired every $30 \mathrm{~s}$ for at least $3 \mathrm{~h}$ with a Zeiss Axiovert 405M microscope using a heated $100 \times$ oil-immersion objective. Growth cones were chosen that extended straight into the spot rim with characteristic dystrophic morphology. Neurons were observed for $30 \mathrm{~min}$ to determine baseline behavior before the addition of inhibitors or enzymes as well as macrophages $(n=6$ for all groups except for embryonic time-lapse imaging for which $n=3$ ). Growth cones were observed for 150 min after enzyme or inhibitor addition. For ChABC studies, $0.1 \mathrm{U} / \mathrm{ml}$ ChABC (Seikagaku) was added through the hole in the dish after $30 \mathrm{~min}$ of baseline imaging. For MMP studies, GM6001 (50 $\mu \mathrm{m})$, MMP-9 (50 nm), or MMP-2 inhibitors (10 $\mu \mathrm{m}$, all from Calbiochem) in DMSO (Sigma-Aldrich) were added after 

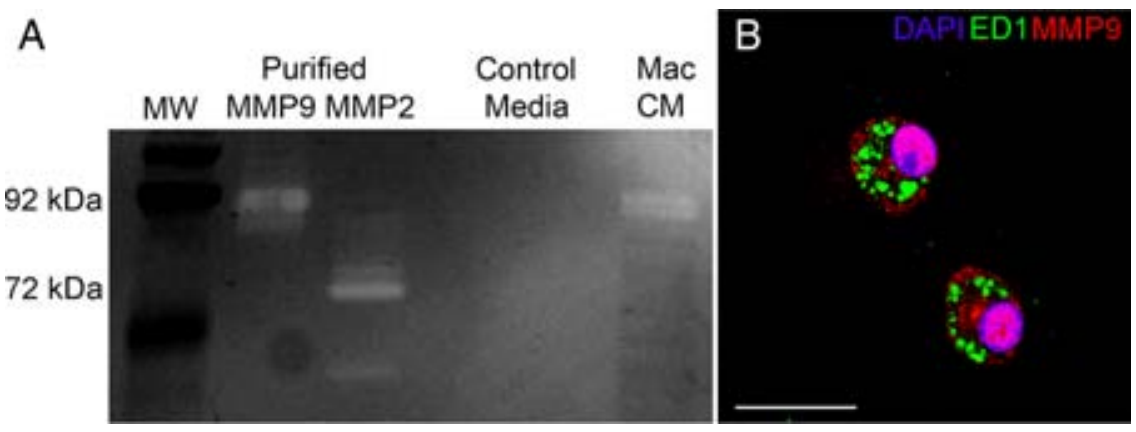

Figure 2. Macrophages express and secrete MMP-9 in vitro. A, Gelatin zymographic analysis of macrophage-conditioned media. Zymogram gel indicates the presence of the active form of MMP-9 at $97 \mathrm{kDa}$ in macrophage-conditioned media, but not in nonconditioned control media. There is no evidence of MMP-2 in either macrophage-conditioned or control media. Purified MMP- 2 and MMP-9 were used as positive controls. $\boldsymbol{B}$, Confocal image $(100 \times)$ of NR8383 macrophages cultured on an inverse spot gradient of proteoglycan and laminin. Immunocytochemistry reveals that activated macrophages, as determined by ED-1 expression (green), contain MMP-9 protein (red). Scale bar: $\boldsymbol{B}, 25 \mu \mathrm{m}$.
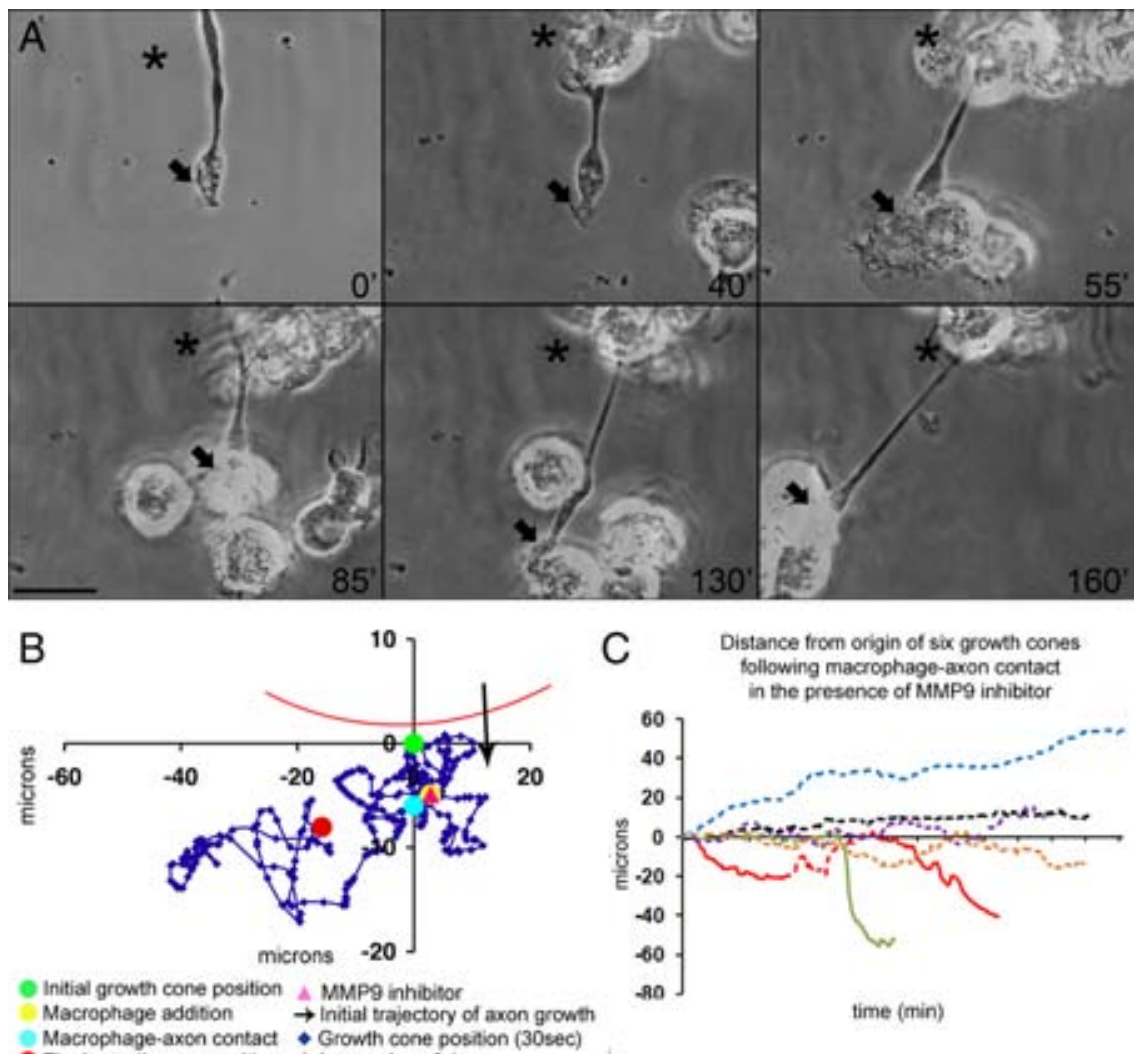

( Mrowh cone position (30sec)

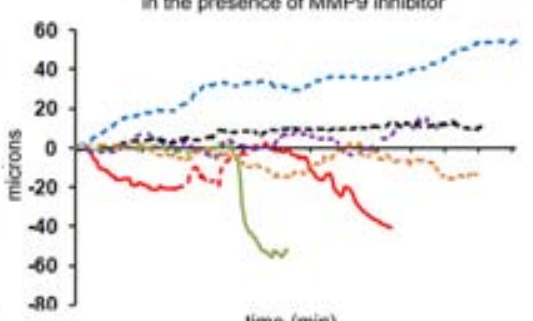

time (min)

Figure 3. Dystrophic adult dorsal root ganglion axons on an inhibitory proteoglycan gradient persist following macrophage contact in the presence of an MMP-9 inhibitor. A, Six-panel montage of a time-lapse movie of NR8383 macrophages and DRG neurons arranged as stated in the Figure 1 legend. Macrophages and MMP-9 inhibitor were added following a 30 min period of observation, and contact occurred at $38 \mathrm{~min}$. Macrophages can be seen physically interacting with the axon at $55 \mathrm{~min}, 85 \mathrm{~min}$, and $130 \mathrm{~min}$ and by $160 \mathrm{~min}$ have pulled the growth cone to the far left of the panel, but no retraction is observed. The entire movie can be viewed as supplemental Movie 2 (available at www.jneurosci.org as supplemental material). $\boldsymbol{B}$, Positional graph indicating the location of the growth cone for the entire time-lapse movie shown in $\boldsymbol{A}$. C, Distance from the origin of six dystrophic axons on the aggrecan/laminin spot gradient in the presence of an MMP-9 inhibitor following contact with macrophages. A dashed line indicates the buffer zone of normal dynamic growth cone extension and collapse and a solid line indicates a long-distance retraction. Each tick mark on the $x$-axis represents $5 \mathrm{~min}$. Scale bar: $A, 20 \mu \mathrm{m}$.

30 min of imaging. Following time-lapse imaging, DRG cultures were fixed in 4\% PFA and immunostained with anti B-tubulin-type III (1:500; Sigma-Aldrich), anti-chondroitin sulfate (CS-56, 1:500, Sigma-Aldrich), 2 B 6 (1:500; Seikagaku), ED-1 (1:500; Millipore Bioscience Research Reagents), and incubated with appropriate secondary antibodies (Invitrogen).
We tracked and charted the behavior of growth cones in our in vitro assay with MetaMorph software. Due to the dynamic nature of growth cones in the inhibitory rim and especially after macrophage contact, we set parameters to accurately reflect the occurrence of large-scale retractions, as opposed to baseline extension and short distance collapse. We considered such a retraction to be at least $25 \mu \mathrm{m}$ in the direction opposite to the original direction of growth. When retractions occur, they are represented as solid lines on the graphs and can occur above or below the $x$-axis.

Total internal reflection fluorescence imaging. Adult DRG neurons were plated on a substrate of proteoglycan and laminin spots as described above on glass bottom dishes (MatTek Corporation) for $2 \mathrm{~d}$ before fixation. Cells were visualized with Phalloidin conjugated to Alexa 594 (Invitrogen) and $\alpha 1 \beta 1$ integrin (1:500; Millipore Bioscience Research Reagents) and the appropriate secondary antibody (Invitrogen) and remained in an aqueous environment throughout the process. Cells were imaged on a Zeiss Axiovert 200M microscope using objectivebased total internal reflection fluorescence (TIRF) with an alpha Plan-Fluar $100 \times$ objective, NA 1.45.

Gelatin-substrate zymography. Conditioned F-12K media from NR8383 macrophages was collected and subjected to gelatin zymography. To prepare the macrophages for conditioned media collection, cells were incubated with $0.5 \%$ trypsin/EDTA (Sigma-Aldrich), washed three times, and plated in uncoated tissue culture flasks at a density of $1.0 \times 10^{6} / \mathrm{ml}$ in serum-free F-12K. After $24 \mathrm{~h}$, conditioned media was removed from the flasks, centrifuged, and passed through a $0.22 \mu \mathrm{m}$ filter (Millipore) to remove debris. Macrophage-conditioned media was then concentrated $50 \times$ using $\mathrm{Mi}-$ crocon YM-3 centrifugal concentration tubes (Millipore). Zymography was performed as previously described (Krekoski et al., 2002). Briefly, SDS-polyacrylamide gels $(7.5 \%)$ were prepared in which $0.1 \%$ gelatin was incorporated during polymerization. Enzyme samples were mixed with $2 \times$ nonreducing sample buffer and loaded onto the lanes of the gel and subjected to electrophoresis at $4^{\circ} \mathrm{C}$ using a BioRad mini gel apparatus. Samples $(50 \mu \mathrm{g})$ of purified MMP-2 and MMP-9 were included on the gel as a positive control (Millipore Bioscience Research Reagents). The gel was briefly rinsed in distilled $\mathrm{H}_{2} \mathrm{O}$ and washed with $2.5 \%$ Triton X-100 three times. The Triton X-100 was removed with three 5 min water washes. The gel was incubated for $21 \mathrm{~h}$ at $37^{\circ} \mathrm{C}$ in buffer containing $50 \mathrm{~mm}$ Tris- $\mathrm{HCl}, 5 \mathrm{~mm} \mathrm{CaCl} 2$, and $0.02 \% \mathrm{NaN}_{2}$. The gel was stained for $1 \mathrm{~h}$ with 0.05\% Coomassie Brilliant Blue and destained in solution without Coomassie Blue until clear proteolytic bands appeared. The identity of the MMP was determined based on comigration with recombinant MMP-9 as well as molecular weight standards.

Dorsal column crush lesion model. All animal procedures were performed in accordance with the guidelines and protocols of the Animal Resource Center at Case Western Reserve University. Adult female Sprague Dawley rats (250-300 g) were anesthetized with inhaled isoflu- 
rane gas ( $2 \%$ in oxygen) for all surgical procedures. The dorsal aspect of the C8 spinal cord segment was exposed by performing a T1 laminectomy. A durotomy was made bilaterally $0.75 \mathrm{~mm}$ from midline with a 30 gauge needle. A dorsal column crush lesion was then made by inserting Dumont \#3 jeweler's forceps into the dorsal spinal cord at C8 to a depth of $1.0 \mathrm{~mm}$ and squeezing the forceps, holding pressure for 10 seconds. This crushing procedure was then repeated two additional times and completion of the lesion was verified by observation of white matter clearing. Gel film was placed over the lesion to cover the holes in the dura. The muscle layers were closed with 4-0 nylon sutures, and the skin was closed with surgical staples. Upon completion of the surgery, animals received Marcaine $(1.0 \mathrm{mg} / \mathrm{kg})$ subcutaneously along the incision as well as buprenorphine $(0.1 \mathrm{mg} / \mathrm{kg})$ intramuscularly. Animals were kept warm postoperatively with a heating lamp during recovery from anesthesia and allowed access to food and water ad libitum. Animals were killed at 2, 4, 7, 14, or $28 \mathrm{~d}$ after lesion $(n=$ 3 per group).

Conditioning lesion. Adult female Sprague Dawley rats were anesthetized as described above. An incision was made into the right thigh, and the sciatic nerve was exposed. The nerve was crushed three times with jeweler's forceps for $10 \mathrm{~s}$ each, proximal to the tibial/ peroneal division. The muscle was closed using 4-0 nylon sutures, and the skin was closed with staples. After $7 \mathrm{~d}$, rats $(n=3$ for each group) received dorsal column crush lesions as described above. From additional animals, L4, L5, and L6 conditioned (right) and unconditioned control (left) DRGs were removed, dissociated, and plated as described above for use in time-lapse microscopy experiments.

Axon labeling. Two days before perfusion, the dorsal columns were labeled unilaterally with Texas Red-conjugated 3000 MW dextran. Briefly, the sciatic nerve of the right hindlimb was exposed and isolated as described for the conditioning lesion procedure above. Sciatic nerves were crushed with Dumont \#3 forceps for $10 \mathrm{~s}$ and repeated two additional times. A hole was made in the epineurium with a 30 gauge needle and then $1.0 \mu \mathrm{l}$ of 3000 MW dextran-Texas Red $10 \%$ in sterile water was injected via a Hamilton syringe into the sciatic nerve at the crush site. The muscle layers were closed with 4-0 nylon sutures and the skin with surgical staples. Upon closing of the incision, animals received Marcaine $(1.0 \mathrm{mg} / \mathrm{kg})$ subcutaneously along the incision as well as buprenorphine $(0.1 \mathrm{mg} / \mathrm{kg})$ intramuscularly. Postoperatively, animals were kept warm with a heating lamp during recovery from anesthesia and allowed access to food and water ad libitum. Animals were killed $2 \mathrm{~d}$ following labeling with an overdose of isoflurane and perfused with PBS followed by $4 \%$ PFA. Tissue was harvested and postfixed in $4 \%$ PFA and processed for immunohistochemistry.

Immunohistochemistry. Tissue was postfixed overnight in 4\% PFA, submersed in $30 \%$ sucrose overnight, frozen in OCT mounting media, and sectioned $(20 \mu \mathrm{m})$ with a cryostat. Tissue sections were then stained with anti-GFAP (Accurate Chemical and Scientific), anti-ED-1 (Millipore) with AlexaFluor-405 or AlexaFluor-488 (Invitrogen) secondary antibodies respectively, and then imaged on a Zeiss Axiovert 510 laserscanning confocal microscope.

In vivo quantification of axonal retraction. Quantification of axonal retraction was performed as described previously (Horn et al., 2008). Briefly, three consecutive sections starting at a depth of $200 \mu \mathrm{m}$ below the dorsal surface of the spinal cord per animal were imaged and analyzed per animal to quantify axonal retraction. The lesion center was determined by the characteristic GFAP and ED-1 staining patterns and the distance between the end of the labeled axons and the center of the lesion was measured using Zeiss LSM 5 Image Browser software. The measurements from all sections from all animals in a group were combined and averaged to yield the average distance of retraction per time point.

Statistical analysis. Data were analyzed by one- or two-way ANOVA and Tukey's post hoc test with Minitab 15 software.

\section{Results}

\section{Dystrophic growth cones retract extensively after contact with macrophages in an in vitro model of the glial scar}

Previous studies from our laboratory demonstrated the direct role of activated macrophages in axonal retraction following dorsal column crush injury (Horn et al., 2008). Adult sensory neurons form dystrophic endbulbs, the hallmark of regeneration failure, in an in vitro model of the glial scar (Ramon y Cajal, 1928; Tom et al., 2004). The substrate in our model consists of opposing gradients of the growth-promoting protein laminin and the inhibitory proteoglycan aggrecan. We have previously shown that activated primary macrophages induce axonal retraction in vitro equivalent to that of NR8383 macrophages (Horn et al., 2008). Following a $30 \mathrm{~min}$ period of baseline observation, NR8383 macrophages were added to the cultures and their interactions with dystrophic axons were mon- 

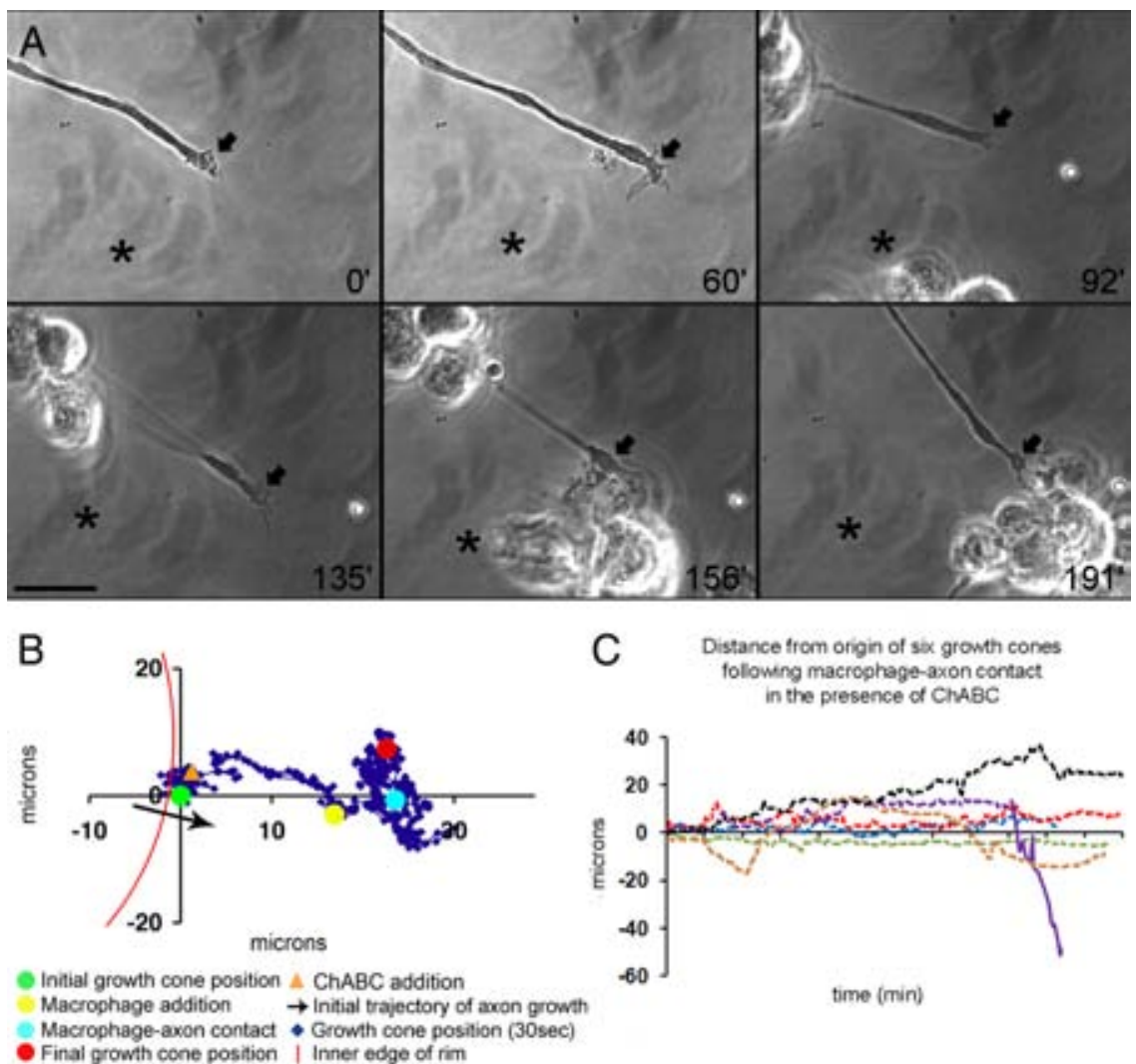

Figure 5. Dystrophic adult dorsal root ganglion axons on an inhibitory proteoglycan gradient treated with ChABC persist after macrophage contact. $A$, Six-panel montage of a time-lapse movie of NR8383 macrophages and DRG neurons arranged as stated in the Figure 1 legend. ChABC $(0.1 \mathrm{U} / \mathrm{ml})$ was added to the dish following a 30 min period of observation and growth cones were observed for positional and morphological changes for $30 \mathrm{~min}$ before macrophage addition. Macrophage contact occurred at 62 $\mathrm{min}$, and the axon did not retract by the end of time-lapse imaging at $180 \mathrm{~min}$. $\boldsymbol{B}$, Positional graph indicating the location of the growth cone for the entire time-lapse movie shown in $\boldsymbol{A}$. Note the forward movement of the growth cone into the rim after $\mathrm{ChABC}$ addition. $C$, Distance from the origin of six dystrophic axons on the aggrecan/laminin spot gradient treated with ChABC following contact with macrophages. A dashed line indicates the buffer zone of normal dynamic growth cone extension and collapse and a solid line indicates a long-distance retraction. Each tick mark on the $x$-axis represents $5 \mathrm{~min}$. Scale bar: $\boldsymbol{A}, 20 \mu \mathrm{m}$.

itored (Fig. 1; supplemental Movie 1, available at www.jneurosci. org as supplemental material). Direct cell-cell contact between the macrophage and axon of the dystrophic neuron led to extensive retraction. The contacts between the macrophage and axon are so strong that they are maintained as the axon retracts over considerable distances (Fig. $1 A, B$; supplemental Movie 1, available at www.jneurosci.org as supplemental material). On average, axons retract at a velocity of $2 \mu \mathrm{m} / \mathrm{min}$, but retraction can occur in spurts of up to $18 \mu \mathrm{m} / \mathrm{min}$. In several cases we observed a retraction fiber remaining attached to the substrate where the growth cone had been even as the axon retracted a long distance (Fig. $1 A)$. Macrophage contact induced retraction of dystrophic axons in an in vitro model of the glial scar $100 \%$ of the time (Fig. 1C). We have observed that during macrophage-induced retraction of axons there is a transition between a dystrophic, vesicle-filled growth cone and a retraction club. The dystrophic growth cone actively turns over membrane and attempts to extend, while the tightly compacted retraction club does not (supplemental Movie 1 , available at www.jneurosci.org as supplemental material).

\section{Macrophages express and secrete MMP-9}

While it is clear that there is a strong physical component to macrophage-mediated axonal retraction, we hypothesized that a protease could be responsible for locally dislodging a dystrophic axon from the substrate, subsequently causing it to retract. One class of proteases expressed by macrophages is the MMPs, which have been implicated in various processes after injury in vivo (Noble et al., 2002; Goussev et al., 2003; Larsen et al., 2003; Hsu et al., 2006). Previous work has established that MMP-9 plays a key role in inflammation within the first $3 \mathrm{~d}$ after spinal cord injury (Noble et al., 2002) and that MMP-9 is produced by macrophages (Larsen et al., 2003). Activated primary (data not shown) and NR8383 macrophages were immunoreactive for MMP-9 in culture (Fig. 2 B). To test for the presence of MMPs, we monitored MMP secretion from NR8383 macrophages into F-12K media using gelatin zymography, which allows for the detection of gelatinases such as MMP-2 and MMP-9. We performed zymography using macrophage-conditioned media and demonstrated MMP-9 gelatinolytic activity at $92 \mathrm{kDa}$, but not MMP-2 activity (Fig. $2 \mathrm{~A}$ ).

\section{Broad-spectrum inhibition of matrix metalloproteinases prevents macrophage-mediated axonal retraction} The net effect of the increase in MMPs seems to be negative, as their inhibition with general metalloproteinase inhibitors, such as GM6001, attenuates vascular events and improves outcome over the first $3 \mathrm{~d}$ after spinal cord injury in rodents (Noble et al., 2002; Wang and Tsirka, 2005). We hypothesized that a general inhibitor of MMPs could prevent macrophage-induced axonal retraction in our in vitro model. We added GM6001, a nonspecific inhibitor of MMPs, and NR8383 macrophages to the DRG culture following a $30 \mathrm{~min}$ period of observation. Macrophage motility and morphology were unaltered in the presence of the inhibitor, and contacts between macrophages and neurons were still extensive and long lasting. In the presence of the inhibitor, growth cones only retracted $33 \%$ of the time following direct macrophage contact (supplemental Fig. 1, available at www. jneurosci.org as supplemental material, and data not shown). In the absence of macrophages, GM6001 had no effect on growth cone morphology and did not induce extension or other notable changes in axonal motility. These data implicated MMPs as the proteases mediating macrophage-induced axonal retraction.

Inhibition of MMP-9, but not MMP-2, prevents macrophagemediated retraction of dystrophic axons

MMP-9 is produced by activated microglia/macrophages (Larsen et al., 2003). Because macrophages express and secrete MMP-9, we asked whether a specific inhibitor to MMP-9 would also prevent macrophage-mediated axonal retraction. In the presence of MMP-9 inhibitor, dystrophic axons retracted 33\% of the time ( $n=4 / 6)$ despite extensive contact between the neurons and macrophages and lifting of the axon from the substrate (Fig. $3 A-C$; supplemental Movie 2, available at www.jneurosci.org as 
supplemental material). Physical manipulation, including stretching and pulling of the axon, is usually insufficient to dislodge the dystrophic growth cone from the substrate. It is important to note that when retractions were observed in the presence of the MMP-9 inhibitor, they were different from macrophage-induced retractions in control cultures. These retractions took place only after extensive physical interactions with active macrophages, and axons retracted slowly and often stabilized before retraction was complete.

Because macrophage-conditioned media lacks MMP-2, we sought to confirm that inhibition of MMP-2 would not prevent macrophage-mediated axonal retraction. As anticipated, growth cones contacted by macrophages in the presence of a specific MMP-2 inhibitor retracted $100 \%$ of the time $(n=6 / 6)$ (Fig. 4A-C; supplemental Movie 3, available at www.jneurosci.org as supplemental material). These data demonstrate that macrophage-induced axonal retraction can be prevented in vitro by the addition of an inhibitor of MMP-9, but not MMP-2.

Substrate cleavage by MMP-9 could be one of several potential mechanisms by which macrophages detach axons from the substrate, ultimately leading to retraction. To determine the effect of these macrophage-secreted MMPs on the substrates of the spot gradient, we fixed cultures after the time-lapse movie and stained for CS-56 and laminin. We did not observe substrate digestion after this $3 \mathrm{~h}$ time period, the length of time of a typical time-lapse movie. Dystrophic growth cones in the inhibitory proteoglycan rim express $\alpha 1$ integrin as visualized by TIRF microscopy, a widefield technique used to excite fluorophores within a thin optical slice of a cell-substrate interface (supplemental Fig. 2, available at www.jneurosci.org as supplemental material). $\alpha 1$ integrins at the cell-substrate interface likely bind the low amount of laminin present and could be another candidate for MMP cleavage.

\section{Digestion of proteoglycan with chondroitinase $\mathrm{ABC}$ prevents axonal retraction}

Our results led us to ask whether other treatment paradigms that have been thought to increase regeneration prevent macrophageinduced axonal retraction. Numerous studies have shown that digestion of inhibitory CSPGs with the bacterial enzyme ChABC increases axonal growth both in vitro and in vivo (Bradbury et al., 2002; Steinmetz et al., 2005). In these experiments, we imaged dystrophic growth cones for $30 \mathrm{~min}$ before adding ChABC to the culture and then for an additional 30 min before adding macrophages. ChABC application to the time-lapse dish induced a noticeable morphological change in the growth cones, which became more dynamic and advanced further into the rim of the spot (Figs. 5A, $B, 6 C$ ). Growth cone morphology after $\mathrm{ChABC}$ addition varied widely. Growth cones could extend more filopodia, lamellipodia, or simply remain a bulbous structure even as forward progress was made. When NR8383 macrophages were added, the retraction phenomenon was prevented $(n=4 / 6$,
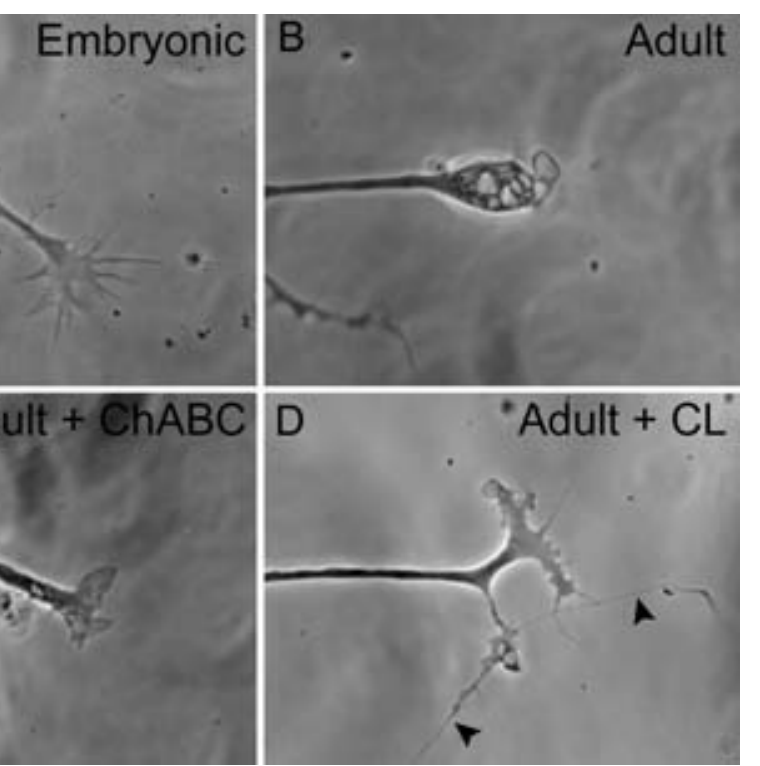

Figure 6. Comparison of embryonic, dystrophic, ChABC treated, and conditioned adult growth cones. $A$, Growth cone of an c day 16 dorsal root ganglion neuron on an inverse spot gradient of growth-promoting molecule laminin and the

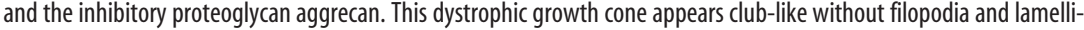
ganglion neuron on an inverse spot gradient of growth-promoting molecule laminin and the inhibitory proteoglycan aggre. It is important to note that both substrate modification $(\boldsymbol{C})$ and conditioning lesion $(\boldsymbol{D})$ induce growth cone structures that are reminiscent of embryonic neurons. Scale bar: $20 \mu \mathrm{m}$.

Fig. 5A-C). Once again, direct cell-cell contact between macrophages and growth cones did occur, but the axons did not retract and the growth cones often appeared to convert from a state of dystrophy to more active growth.

\section{Embryonic neurons are resistant to macrophage attack in vitro}

Embryonic neurons have a greater propensity to regenerate than their adult counterparts, and can readily adapt to inhibitory conditions via upregulation of integrin receptors for growth-promoting molecules (Condic, 2001). Interestingly, rat embryonic day 16 DRG growth cones do not become dystrophic and also extend greater numbers of filopodia than adult growth cones (Fig. 6A). All embryonic neurons take on this morphology. Like their adult counterparts, embryonic axons enter the rim and stall while still attempting forward growth, but cannot cross the rim and do not tend to turn. Embryonic growth cones actively extend and retract lamellipodia and filopodia and maintain a strong attachment to the inhibitory rim. Embryonic neurons were also more resistant to macrophage attack as they maintained a strong attachment to the substrate at the growth cone (data not shown), suggesting that the maturation state of the neuron is critical to the outcome of macrophage-mediated axonal retraction.

\section{Preconditioning adult neurons reduces macrophage- mediated axonal retraction}

To determine whether CL-induced stimulation of sensory neuron intrinsic growth potential would also reduce macrophageinduced axonal retraction we conditioned dorsal root ganglion neurons with a sciatic nerve crush $7 \mathrm{~d}$ before harvest (Steinmetz et 

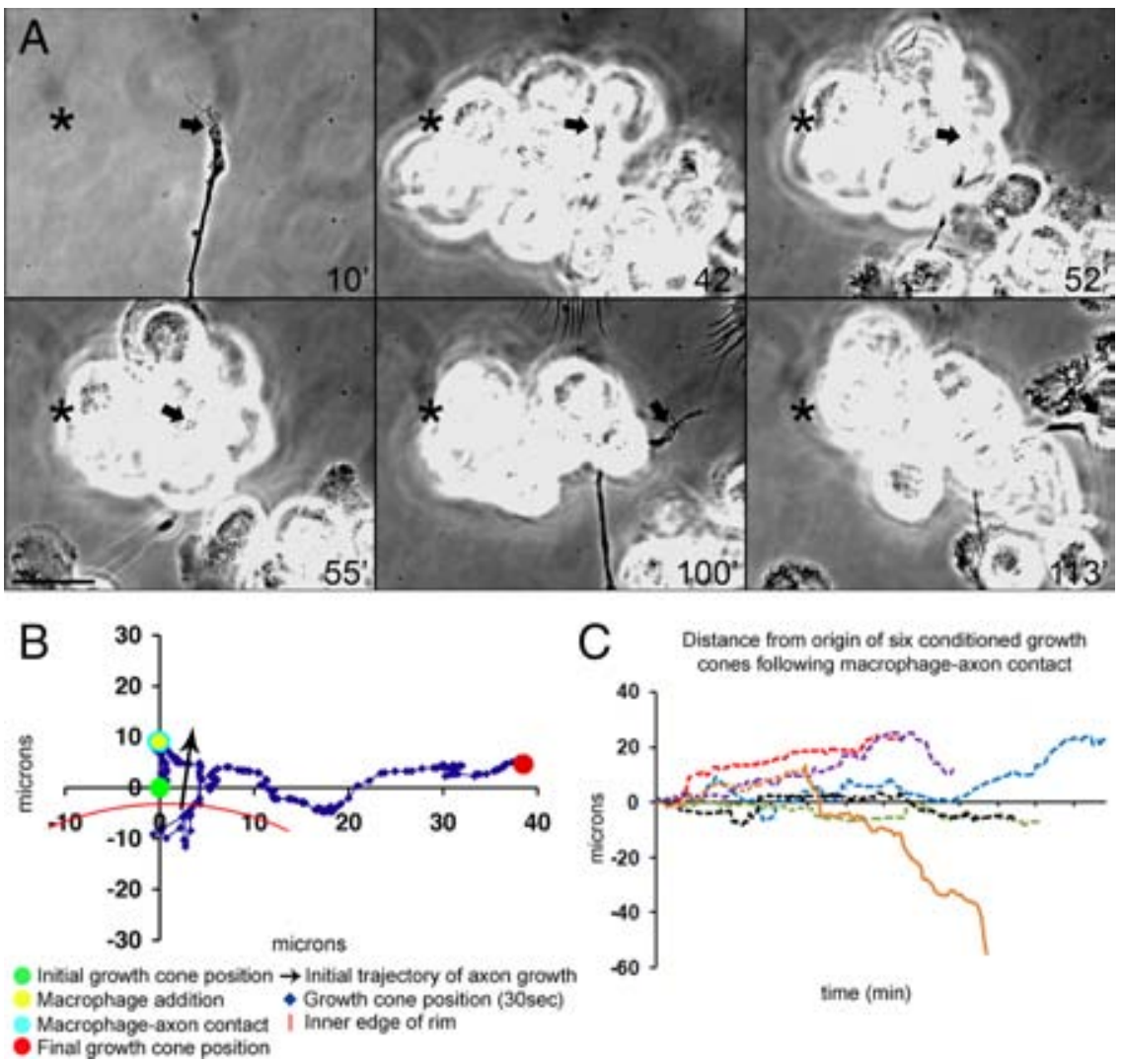

Figure 7. Conditioned adult dorsal root ganglion axons on an inhibitory proteoglycan gradient persist after macrophage contact. $A$, Six-panel montage of a time-lapse movie of NR8383 macrophages and conditioned DRG neurons arranged as stated in the Figure 1 legend. Macrophage contact occurred at $42 \mathrm{~min}$ and the axon did not retract by the end of time-lapse imaging. The entire movie can be viewed as supplemental Movie 4 (available at www.jneurosci.org as supplemental material). $\boldsymbol{B}$, Positional graph indicating the location of the growth cone for the entire time-lapse movie shown in $\boldsymbol{A}$. Note the lateral movement of the growth cone along the rim. C, Distance from the origin of six axons of conditioned neurons on the aggrecan/laminin spot gradient following contact with macrophages. A dashed line indicates the buffer zone of normal dynamic growth cone extension and collapse, and a solid line indicates a long-distance retraction. Each tick mark on the $x$-axis represents $5 \mathrm{~min}$. Scale bar: $A, 20 \mu \mathrm{m}$.

al., 2005). Unconditioned DRGs become dystrophic in response to the potently inhibitory gradient while conditioned DRGs typically display more dynamic behaviors. Growth cones from animals that received a CL of the sciatic nerve $7 \mathrm{~d}$ prior are considerably more motile on the spot rim, compared with dystrophic growth cones, and exhibit turning behaviors and increased filopodial extension (Fig. 6B,D). Filopodia extending from conditioned axons were often extremely long and appeared to be seeking a more favorable substrate. Dystrophic growth cones can extend short filopodia, but they are fewer in number and not as dynamic. We imaged conditioned growth cones for $30 \mathrm{~min}$ before adding macrophages. Axons continued to struggle despite repeated macrophage attacks and often engaged in abortive turning behaviors. Growth cones maintained strong adhesion to the substrate regardless of the macrophage manipulation of the axon and avoided retraction $(n=5 / 6)$ (Fig. $7 A-C$; supplemental Movie 4, available at www.jneurosci.org as supplemental material).

\section{A conditioning lesion prevents axonal dieback in vivo}

CLs have been purported to promote regeneration of axons farther into and even beyond a lesion, into a region containing predominantly ED-1+ cells (Neumann and Woolf, 1999; Neumann et al., 2005). We hypothesized that interactions between macrophages and growth cones could limit regrowth and that conditioning of sensory axons might enable the struggling axons to withstand macrophage attack. We examined sensory axon retraction after acute and chronic dorsal column crush injury in the presence or absence of a CL. In the CL group, adult female Sprague Dawley rats received a sciatic nerve crush $7 \mathrm{~d}$ before spinal cord injury. On day 7 , the dorsal columns were crushed at spinal level C8, and sensory axons were traced from the right sciatic nerve with dextran-Texas Red. Spinal cord tissue was collected at $2,4,7,14$, and $28 \mathrm{~d}$ after injury, and axonal retraction was measured as described previously (Horn et al., 2008). In animals that received a CL, injured axons retracted significantly less than control, unconditioned axons (Fig. $8 G)$. CL did not prevent the intrinsic, macrophage-independent early phase of axonal retraction at day $2(-387 \mu \mathrm{m} \pm$ 29.5 vs $-241 \mu \mathrm{m} \pm 100.5$ ) (Fig. $8 A, B, G$ ). By day 7 , when macrophages have infiltrated the lesion in large numbers, control axons have retracted significantly further from the lesion center $(-615 \mu \mathrm{m} \pm 119.4$ vs $-346 \mu \mathrm{m} \pm$ 93.1) (Fig. $8 C, D, G$ ). On day 28, conditioned neurons persist within $400 \mu \mathrm{m}$ of the lesion epicenter $(-706 \mu \mathrm{m} \pm 194.9 \mathrm{vs}-398 \mu \mathrm{m} \pm 49.5)$ (Fig. $8 E-G$ ). In addition to conditioned axons persisting closer to the lesion center, the spread of the fiber front was notably wider in animals receiving a CL. We observed no significant difference in macrophage infiltration in control versus conditioned spinal cord sections (Fig. $8 \mathrm{H}$ ); therefore, conditioned axons were subject to the same numbers of macrophages as control axons. These data suggest that conditioned axons do not retract to the same degree as control axons. Instead, they appear to persist in the lesion despite macrophage infiltration.

\section{Discussion}

In the present study, we used our in vitro model of the glial scar to determine the ability of previously identified regenerationpromoting strategies to prevent macrophage-induced retraction of dystrophic adult sensory neurons. We have demonstrated that protease inhibition, substrate modification, and stimulation of the intrinsic growth capacity of adult DRG neurons can all improve the outcome of macrophage attack of dystrophic axons in vitro. Our results suggest that treatment paradigms that have been thought to successfully promote neuronal regeneration following spinal cord injury function in part through inhibition of macrophage-induced axonal retraction as opposed to exerting their influence solely by frank regeneration.

We have determined that specific inhibition of MMP-9, but not MMP-2, prevented macrophage-mediated retraction despite extensive physical manipulation of the axon in vitro. The expression of several MMPs is upregulated following spinal cord injury, and zymography of lesioned tissues shows that MMP-2 and MMP-9 are active in areas containing regenerating neurites (Du- 

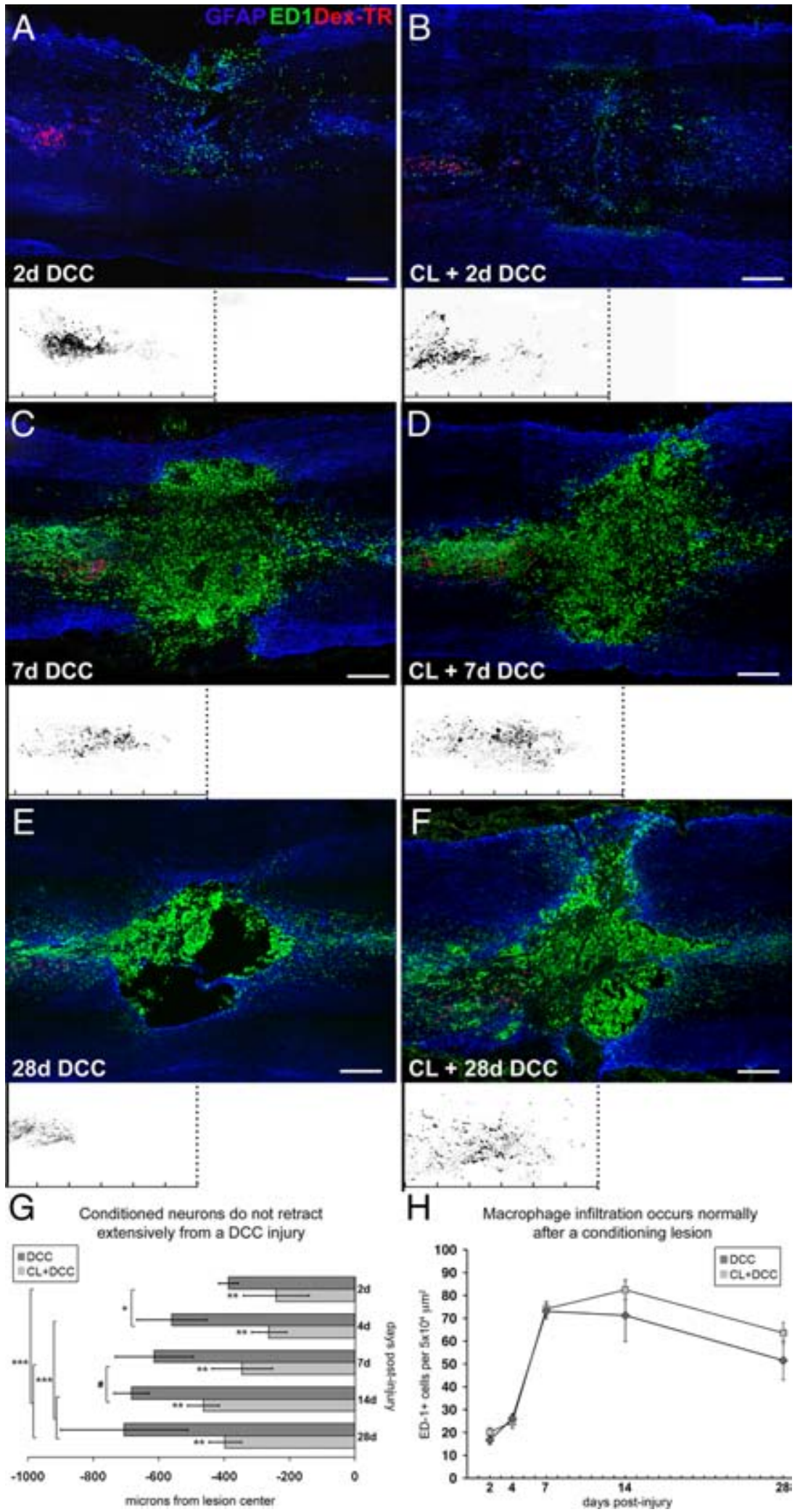

Figure 8. Conditioned neurons persist close to the lesion center after spinal cord injury. $\boldsymbol{A}-\boldsymbol{F}$, Confocal montages $(10 \times)$ of longitudinal sections of animals receiving a dorsal column crush (DCC) spinal cord injury alone or a dorsal column crush spinal cord injury $7 \mathrm{~d}$ after receiving a sciatic crush conditioning lesion $(\mathrm{CL}+\mathrm{DCC})$. Caudal is on the left side of the image and rostral is on the right. Dorsal root ganglion neuron are labeled with Texas Red-conjugated dextran 3000 MW (Dex-TR) (red) and ED-1+ macrophages/microglia (green) and GFAP + astrocytes (blue). Below each confocal image are superimposed fiber tracings of three sections from a representative animal for each time point. Dotted lines represent the lesion center, and ruler tick marks are $200 \mu \mathrm{m}$ each. $\boldsymbol{A}$, At $2 \mathrm{~d}$ after DCC, sensory axons of the dorsal columns have retracted a short distance from the center of the lesion, determined by GFAP + astrocytes (blue). Few ED-1+ macrophages/microglia (green) are present in the lesion at this time. $\boldsymbol{B}, A$ At chossoy et al., 2001). The correlation between the spatiotemporal expression of MMPs and the progression of macrophageinduced retraction indicates that MMP-9 could be mediating this detrimental process in vivo. MMP-9 knock-out mice exhibit a decrease in blood-spinal cord barrier disruption, decreased inflammation, and significant locomotor recovery, and similar results were obtained using a pharmacological inhibitor of MMPs (Noble et al., 2002). Interestingly, methylprednisolone, the only Food and Drug Administration-approved therapy for spinal cord injury, suppresses MMP-9 following spinal cord injury (Xu et al., 2001). Minocycline, another purported regeneration promoting drug, inhibits MMP activity via inhibition of MMP-9 mRNA transcription and protein activity (Yao et al., 2004). Our data identify macrophageassociated MMP-9 as a critical component of the pathway ultimately inducing neuronal retraction and provide insight into the mechanism underlying axonal dieback. A significant decrease in macrophageinduced axonal retraction improves the starting position of the axon within the environment of the spinal cord lesion and may have the added benefit of rescuing collateral branches.

While we have implicated macrophageassociated MMP-9 as a negative influence on axon regeneration in our in vitro model, there are other MMPs as well as other proteases acting in the lesion environment. Thus, MMP-9 may not be the only protease involved with the dieback

$2 \mathrm{~d}$ after $\mathrm{CL}+\mathrm{DCC}$, sensory axons have retracted a short distance from the lesion center, similar to the level observed in DCC animals. C, At $7 \mathrm{~d}$ after DCC, numerous ED-1+ phagocytic cells are present within the lesion and sensory axons have retracted a significant distance from the lesion center. $\boldsymbol{D}$, At $7 \mathrm{~d}$ after $C L+D C C$, fibers are still in close proximity to the center of the lesion despite the presence of numerous ED-1+ phagocytic cells. $\boldsymbol{E}$, At $28 \mathrm{~d}$ after DCC, axons have retracted a considerable distance from the lesion center. $\boldsymbol{F}$, At $28 \mathrm{~d}$ after DCC plus conditioning lesion 1 week before $D C C$ lesion, axons persist close to the lesion center despite the presence of numerous ED-1+ cells, and the fiber front is wider than in control DCC animals. $\mathbf{G}$, Quantification of axonal retraction from the center of the lesion over time. All days are significantly different from one another by one-way ANOVA, $F_{(4,440)}=28.71$. Day 2 is significantly different from days $4,7,14$, and 28 ; day 4 is significantly different from days 14 and 28; day 7 is significantly different from day 14 by Tukey's post hoc test, ${ }^{\#} p<0.01$, ${ }^{*} p<0.005$, ${ }^{* * *} p<0.00005$. Control and conditioning lesion groups are significantly different from one another (one-way ANOVA, $F_{(1.440)}=195.47$, Tukey's post hoc test, ${ }^{* *} p<$ 0.0005). Error bars indicate SEM. $\boldsymbol{H}$, Macrophage infiltration is not significantly different in control and conditioned animals. Scale bars: $A-F, 250 \mu \mathrm{m}$. 
phenomenon in vivo. We cannot ignore the fact that the inhibitors used here are based on the functional group hydroxamate, which chelate a zinc ion from the active site of MMPs, and also have activity against $\mathrm{ADAMs}$ (A disintegrin and metalloproteinases), membraneanchored enzymes that are prevalent in vivo and can also cleave components of the ECM (Yong, 2005). The effect mediated by MMP-9 in vivo could also be indirect, as its primary function may be the activation of additional proteases.

MMPs can degrade ECM molecules such as type IV collagen, laminin, and fibronectin (Chandler et al., 1997; Chintala et al., 2002). We sought to determine whether macrophage-secreted MMPs exerted their dramatic retraction-inducing effects in vitro via substrate digestion that would, in turn, lead to immediate dislocation of integrin-mediated attachments with the substrate. However, immunocytochemistry revealed no visible digestion of either aggrecan or laminin. It is conceivable that local release of MMPs may result in an undetectable amount of ECM digestion as MMPs are typically anchored to the cell membrane, maintaining a high enzyme concentration locally and targeting catalytic activity to specific substrates in the pericellular space (Parks et al., 2004). If MMPs are not acting globally on the substrate itself, it is possible that more focused interactions with extracellular matrix molecules or proteinase-activated receptors (PARs) on the neuronal surface could trigger an intracellular cascade of events leading to retraction (Yong et al., 2001; Noorbakhsh et al., 2003). PAR1 and PAR2 are present on DRG neurons and upon activation, PAR1 initiates G-protein signaling cascades that can lead to increases in intracellular calcium and RhoA-dependent cytoskeletal changes, both of which can induce neurite retraction (Noorbakhsh et al., 2003). As MMPs often act in concert, it is possible that MMP-9 activates another MMP or protease that then acts on the neuron itself. While growth cone contact after macrophage addition is typical, we observed multiple instances in which macrophages never contacted the growth cone itself, instead inducing retraction by attacking the axon shaft. These observations suggest the possibility that a signal could propagate from the macrophage contact point on the axon to the growth cone, ultimately inducing full retraction. A potential signal that could travel rapidly from the axon to the growth cone and mediate axonal retraction is an intracellular calcium wave (Jaffe, 1993; Yamada et al., 2008). $\mathrm{Ca}^{2+}$ waves inhibit neurite extension via the $\mathrm{Ca}^{2+}$-dependent phosphatase calcineurin (Lautermilch and Spitzer, 2000). Calcineurin destabilizes actin microfilaments and disrupts the growth cone actin cytoskeleton, inhibiting neurite outgrowth. Alternatively, macrophages could permeabilize target cells via perforin in a cell-cell contact-dependent manner (Baba et al., 2008), which in combination with another secreted factor could promote cytoskeletal disassembly and retraction.

Previous work from our laboratory showed that the substrate on which a neuron is plated is critical to the outcome of macrophage-neuron interactions, as neurons on uniform laminin substrates do not retract following extensive macrophage contact (Horn et al., 2008). ChABC treatment of our in vitro model of the glial scar allows a small but significant number of axons to cross the inhibitory rim after $5 \mathrm{~d}$ (Steinmetz et al., 2005). In the present study, ChABC application to the culture appeared to enable the growth cone to adhere more strongly to the substrate and resulted in a decrease in frequency of macrophage-induced axonal retraction. In addition to alleviating GAG-mediated inhibition, it is probable that modification of the extracellular matrix with ChABC unmasked laminin present in small quantities in the inhibitory region of the spot assay, providing increased adhesion of the growth cone and ultimately de- creasing the likelihood of axonal retraction. ChABC promotes sprouting of intact and injured projections within the spinal cord (Barritt et al., 2006; Houle et al., 2006; Massey et al., 2006). Previous studies have also suggested that there is an optimal therapeutic time window for ChABC treatment, as acutely treated animals show improved skilled forelimb reaching and greater axon regeneration (García-Alías et al., 2008). Our results indicate that substrate modification can alter the outcome of macrophage attack and suggests that in vivo ChABC treatment of axons within the vicinity of the lesion may additionally augment CNS regeneration by preventing macrophage-induced retraction.

In addition to improving the growth environment of the lesion, enhancing intrinsic neuronal growth capacity with a distal CL can enable regeneration of sensory neurons in the dorsal columns (Richardson and Issa, 1984; Neumann and Woolf, 1999). Our in vitro results indicate that the intrinsic growth state of the neuron can affect the result of macrophage attack. A CL enhances sensory neuron regeneration in part by upregulation of integrin receptors on and increasing responsiveness to laminin (Ekström et al., 2003). The repertoire of integrin receptors on conditioned neurons could enable them to behave more like an embryonic neuron in the face of the inhibitory proteoglycan and low laminin conditions of the spot gradient. Our observations that conditioned sensory neurons display enhanced motility, and a remarkable ability to turn and struggle when faced with an inhibitory gradient of proteoglycan in vitro correlate with previous in vivo observations of struggling axons extending haphazardly into and around the lesion (Neumann and Woolf, 1999).

Previous studies examining the effect of different types of conditioning on the outcome of spinal cord injury have generally focused on long-term time points and determined the position of the axons at these later stages to be the result of regeneration (Neumann and Woolf, 1999; Neumann et al., 2002; Nikulina et al., 2004). Our results indicate that there is a lack of retraction of sensory neurons in vivo as, after the initial intrinsic retraction has taken place, the conditioned fibers remain within $500 \mu \mathrm{m}$ from the lesion on average. Elucidating the underlying cellular and molecular mechanisms of the CL that are critical for axonal regrowth will enable the development of strategies that can promote CNS regeneration without sacrificing the sciatic nerve. Ultimately, attempts to understand pathways involved in regeneration-associated signaling will be critical to potential therapeutic stimulation of growth after CNS injury (Snider et al., 2002; Ertürk et al., 2007; Seijffers et al., 2007; Wang et al., 2007).

In this study we turned to established treatments that promote regeneration as targets for our investigation to determine whether they could prevent macrophage-induced retraction. Our results support the hypothesis that MMP-9 inhibition, ChABC treatment, and pretreatment with a CL all facilitate regeneration in part via prevention of axonal dieback. Our model can be used as a screening tool for potential therapeutic interventions and combinatorial approaches to treat CNS injury and attain the critical balance of a permissive growth environment, stimulation of neuronal growth and regulation of inflammation. Elucidating the basic biology underlying macrophage-induced retraction will enable us to better understand the environment of the injured CNS and prevent secondary damage in the days, weeks, and months following spinal cord injury.

\section{References}

Baba T, Iwasaki S, Maruoka T, Suzuki A, Tomaru U, Ikeda H, Yoshiki T, Kasahara M, Ishizu A (2008) Rat CD4+CD8+ macrophages kill tumor 
cells through an NKG2D- and granzyme/perforin-dependent mechanism. J Immunol 180:2999-3006.

Barritt AW, Davies M, Marchand F, Hartley R, Grist J, Yip P, McMahon SB, Bradbury EJ (2006) Chondroitinase ABC promotes sprouting of intact and injured spinal systems after spinal cord injury. J Neurosci 26:10856-10867.

Bradbury EJ, Moon LD, Popat RJ, King VR, Bennett GS, Patel PN, Fawcett JW, McMahon SB (2002) Chondroitinase ABC promotes functional recovery after spinal cord injury. Nature 416:636-640.

Busch SA, Silver J (2007) The role of extracellular matrix in CNS regeneration. Curr Opin Neurobiol 17:120-127.

Chandler S, Miller KM, Clements JM, Lury J, Corkill D, Anthony DC, Adams SE, Gearing AJ (1997) Matrix metalloproteinases, tumor necrosis factor and multiple sclerosis: an overview. J Neuroimmunol 72:155-161.

Chintala SK, Zhang X, Austin JS, Fini ME (2002) Deficiency in matrix metalloproteinase gelatinase B (MMP-9) protects against retinal ganglion cell death after optic nerve ligation. J Biol Chem 277:47461-47468.

Condic ML (2001) Adult neuronal regeneration induced by transgenic integrin expression. J Neurosci 21:4782-4788.

Davies SJ, Goucher DR, Doller C, Silver J (1999) Robust regeneration of adult sensory axons in degenerating white matter of the adult rat spinal cord. J Neurosci 19:5810-5822.

Duchossoy Y, Horvat JC, Stettler O (2001) MMP-related gelatinase activity is strongly induced in scar tissue of injured adult spinal cord and forms pathways for ingrowing neurites. Mol Cell Neurosci 17:945-956.

Ekström PA, Mayer U, Panjwani A, Pountney D, Pizzey J, Tonge DA (2003) Involvement of alpha7betal integrin in the conditioning-lesion effect on sensory axon regeneration. Mol Cell Neurosci 22:383-395.

Ertürk A, Hellal F, Enes J, Bradke F (2007) Disorganized microtubules underlie the formation of retraction bulbs and the failure of axonal regeneration. J Neurosci 27:9169-9180.

Fawcett JW (2006) Overcoming inhibition in the damaged spinal cord. J Neurotrauma 23:371-383.

Filbin MT (2003) Myelin-associated inhibitors of axonal regeneration in the adult mammalian CNS. Nat Rev Neurosci 4:703-713.

García-Alías G, Lin R, Akrimi SF, Story D, Bradbury EJ, Fawcett JW (2008) Therapeutic time window for the application of chondroitinase $\mathrm{ABC}$ after spinal cord injury. Exp Neurol 210:331-338.

Goussev S, Hsu JY, Lin Y, Tjoa T, Maida N, Werb Z, Noble-Haeusslein LJ (2003) Differential temporal expression of matrix metalloproteinases after spinal cord injury: relationship to revascularization and wound healing. J Neurosurg 99:188-197.

Horn KP, Busch SA, Hawthorne AL, van Rooijen N, Silver J (2008) Another barrier to regeneration in the CNS: activated macrophages induce extensive retraction of dystrophic axons through direct physical interactions. J Neurosci 28:9330-9341.

Houle JD, Tom VJ, Mayes D, Wagoner G, Phillips N, Silver J (2006) Combining an autologous peripheral nervous system "bridge" and matrix modification by chondroitinase allows robust, functional regeneration beyond a hemisection lesion of the adult rat spinal cord. J Neurosci 26:7405-7415.

Hsu JY, McKeon R, Goussev S, Werb Z, Lee JU, Trivedi A, NobleHaeusslein LJ (2006) Matrix metalloproteinase-2 facilitates wound healing events that promote functional recovery after spinal cord injury. J Neurosci 26:9841-9850.

Jaffe LF (1993) Classes and mechanisms of calcium waves. Cell Calcium 14:736-745.

Krekoski CA, Neubauer D, Graham JB, Muir D (2002) Metalloproteinasedependent predegeneration in vitro enhances axonal regeneration within acellular peripheral nerve grafts. J Neurosci 22:10408-10415.

Larsen PH, Wells JE, Stallcup WB, Opdenakker G, Yong VW (2003) Matrix metalloproteinase- 9 facilitates remyelination in part by processing the inhibitory NG2 proteoglycan. J Neurosci 23:11127-11135.

Lautermilch NJ, Spitzer NC (2000) Regulation of calcineurin by growth cone calcium waves controls neurite extension. J Neurosci 20:315-325.

Massey JM, Hubscher CH, Wagoner MR, Decker JA, Amps J, Silver J, Onifer SM (2006) Chondroitinase $\mathrm{ABC}$ digestion of the perineuronal net promotes functional collateral sprouting in the cuneate nucleus after cervical spinal cord injury. J Neurosci 26:4406-4414.

Neumann S, Woolf CJ (1999) Regeneration of dorsal column fibers into and beyond the lesion site following adult spinal cord injury. Neuron 23:83-91.

Neumann S, Bradke F, Tessier-Lavigne M, Basbaum AI (2002) Regenera- tion of sensory axons within the injured spinal cord induced by intraganglionic cAMP elevation. Neuron 34:885-893.

Neumann S, Skinner K, Basbaum AI (2005) Sustaining intrinsic growth capacity of adult neurons promotes spinal cord regeneration. Proc Natl Acad Sci U S A 102:16848-16852.

Nikulina E, Tidwell JL, Dai HN, Bregman BS, Filbin MT (2004) The phosphodiesterase inhibitor rolipram delivered after a spinal cord lesion promotes axonal regeneration and functional recovery. Proc Natl Acad Sci U S A 101:8786-8790.

Noble LJ, Donovan F, Igarashi T, Goussev S, Werb Z (2002) Matrix metalloproteinases limit functional recovery after spinal cord injury by modulation of early vascular events. J Neurosci 22:7526-7535.

Noorbakhsh F, Vergnolle N, Hollenberg MD, Power C (2003) Proteinaseactivated receptors in the nervous system. Nat Rev Neurosci 4:981-990.

Parks WC, Wilson CL, López-Boado YS (2004) Matrix metalloproteinases as modulators of inflammation and innate immunity. Nat Rev Immunol 4:617-629.

Popovich PG, Longbrake EE (2008) Can the immune system be harnessed to repair the CNS? Nat Rev Neurosci 9:481-493.

Qiu J, Cai D, Dai H, McAtee M, Hoffman PN, Bregman BS, Filbin MT (2002) Spinal axon regeneration induced by elevation of cyclic AMP. Neuron 34:895-903.

Ramon y Cajal S (1928) Degeneration and regeneration of the nervous system. London: Oxford UP.

Richardson PM, Issa VM (1984) Peripheral injury enhances central regeneration of primary sensory neurones. Nature 309:791-793.

Seijffers R, Mills CD, Woolf CJ (2007) ATF3 increases the intrinsic growth state of DRG neurons to enhance peripheral nerve regeneration. J Neurosci 27:7911-7920.

Silver J, Miller JH (2004) Regeneration beyond the glial scar. Nat Rev Neurosci 5:146-156.

Snider WD, Zhou FQ, Zhong J, Markus A (2002) Signaling the pathway to regeneration. Neuron 35:13-16.

Steinmetz MP, Horn KP, Tom VJ, Miller JH, Busch SA, Nair D, Silver DJ, Silver J (2005) Chronic enhancement of the intrinsic growth capacity of sensory neurons combined with the degradation of inhibitory proteoglycans allows functional regeneration of sensory axons through the dorsal root entry zone in the mammalian spinal cord. J Neurosci 25:8066-8076.

Tester NJ, Howland DR (2008) Chondroitinase ABC improves basic and skilled locomotion in spinal cord injured cats. Exp Neurol 209:483-496.

Tom VJ, Steinmetz MP, Miller JH, Doller CM, Silver J (2004) Studies on the development and behavior of the dystrophic growth cone, the hallmark of regeneration failure, in an in vitro model of the glial scar and after spinal cord injury. J Neurosci 24:6531-6539.

Wang J, Tsirka SE (2005) Neuroprotection by inhibition of matrix metalloproteinases in a mouse model of intracerebral haemorrhage. Brain 128:1622-1633.

Wang W, van Niekerk E, Willis DE, Twiss JL (2007) RNA transport and localized protein synthesis in neurological disorders and neural repair. Dev Neurobiol 67:1166-1182.

Xu J, Kim GM, Ahmed SH, Xu J, Yan P, Xu XM, Hsu CY (2001) Glucocorticoid receptor-mediated suppression of activator protein-1 activation and matrix metalloproteinase expression after spinal cord injury. J Neurosci 21:92-97.

Yamada RX, Sasaki T, Ichikawa J, Koyama R, Matsuki N, Ikegaya Y (2008) Long-range axonal calcium sweep induces axon retraction. J Neurosci 28:4613-4618.

Yao JS, Chen Y, Zhai W, Xu K, Young WL, Yang GY (2004) Minocycline exerts multiple inhibitory effects on vascular endothelial growth factorinduced smooth muscle cell migration: the role of ERK1/2, PI3K, and matrix metalloproteinases. Circ Res 95:364-371.

Yin Y, Cui Q, Li Y, Irwin N, Fischer D, Harvey AR, Benowitz LI (2003) Macrophage-derived factors stimulate optic nerve regeneration. J Neurosci 23:2284-2293.

Yiu G, He Z (2006) Glial inhibition of CNS axon regeneration. Nat Rev Neurosci 7:617-627.

Yong VW (2005) Metalloproteinases: mediators of pathology and regeneration in the CNS. Nat Rev Neurosci 6:931-944.

Yong VW, Power C, Forsyth P, Edwards DR (2001) Metalloproteinases in biology and pathology of the nervous system. Nat Rev Neurosci 2:502-511. 